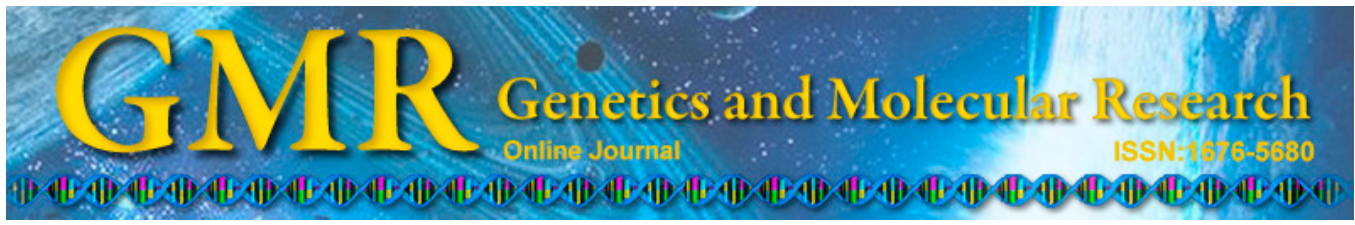

\title{
Genetic diversity of Y-short tandem repeats in chinese native cattle breeds
}

\author{
Y.P. Xin ${ }^{1}$, L.S. Zan'1, Y.F. Liu' ${ }^{2}$ W.Q. Tian ${ }^{3}$, H.B. Wang', G. Cheng1, \\ A.N. Li ${ }^{1}$ and W.C. Yang ${ }^{1}$ \\ ${ }^{1}$ College of Animal Science and Technology, Northwest A\&F University, \\ Yangling, Shaanxi, China \\ ${ }^{2}$ College of Food Engineering and Nutritional Science, \\ Shaanxi Normal University, Xi'an, Shaanxi, China \\ ${ }^{3}$ Yangling Vocational Technical College, Yangling, Shaanxi, China \\ Corresponding author: L.S. Zan \\ E-mail: zanls@yahoo.com.cn
}

Genet. Mol. Res. 13 (4): 9578-9587 (2014)

Received June 21, 2013

Accepted September 22, 2014

Published November 14, 2014

DOI http://dx.doi.org/10.4238/2014.November.14.1

\begin{abstract}
The aim of this study is to use Y-chromosome gene polymorphism method to investigate regional differences in genetic variation and population evolution history of the Chinese native cattle breeds. Six Y-chromosome short tandem repeat (Y-STR) loci (UMN0929, UMN0108, UMN0920, INRA124, UMN2404, and UMN0103) were analyzed using 1016 healthy and heterogenetic males and 90 females of 9 native cattle breeds (Qinchuan, Jinnan, Zaosheng, Luxi, Nanyang, Jiaxian, Dabieshan, Yanbian, and Menggu) in China. Allele frequency and gene diversity were calculated for the various populations. The results indicated that Y-STRs in the 6 loci have polymorphisms and genetic diversity in Chinese cattle populations. The genetic diversity analysis revealed that the Chinese cattle populations have a close genetic relationship. The analysis of INRA124, UMN2404, and UMN0103 loci revealed the original history of Chinese cattle because of which cattle belonging to Bos taurus or Bos indicus could be determined. Interestingly, a declining zebu introgression was displayed
\end{abstract}


from South to North and from East to West in the Chinese geographical distribution, which implied that cattle population from various regions of China had been subjected to somewhat different evolutionary history. This conclusion supported other evidences such as earlier archaeological, historical research, and blood protein polymorphism analysis.

Key words: Cattle; Y-chromosome; Polymorphism; Haplotype; Short tandem repeat

\section{INTRODUCTION}

China has many native cattle breeds. According to the Chinese Cattle Breed Encyclopedia Record, at least 28 types of Chinese cattle have been identified as local fine breeds ( $\mathrm{Li}$ et al., 2013a). In the last half century, many animal scientists were interested in understanding Chinese native cattle genetic construction, origin, and evolution. About 20 years ago, the blood protein polymorphism technology was first used to study the origins and genetic variability of Chinese yellow cattle; this study indicated that Qinchuan cattle has a close genetic relationship with Jinnan cattle, and that they contain Bos taurus cattle blood (Wu, 1988). With advancement in molecular biology techniques, DNA polymorphism has been widely used for studying animal breeding resources.

During the last decade, Y-chromosome-specific markers were used in the studies of human origins because the frequency and geographical distribution of Y-haplotypes provide useful information about migration, sex-specific gene flow, and population relationships (Jorde et al., 2000). DNA Y-chromosome segment (DYS) is a segment of DNA on the Y chromosome that has many nucleotide repeats. These sequences are known as short tandem repeats (STRs) and are used as markers for genealogical DNA testing. The possible variations of repeats at a DYS marker are known as alleles.

Y-STRs are short tandem repeats found on male-specific Y-chromosomes. These coding genes are vital for male sex determination, spermatogenesis, and other male-related functions. The Y-STRs are polymorphisms among unrelated males and are inherited from the paternal line with little change through generations. The non-recombinant Y-chromosome segment dictates the paternally inherited haploid transmission pattern. Y-STRs can be employed to construct highly discriminative Y-haplotypes (Redd et al., 2006). In humans, the polymorphism of Y-STRs has been used in anthropology and forensic genetic studies (Gill et al., 2001). However, information on the Y-STR in farm animals is limited. Giovambattista et al. (2000) analyzed the polymorphism of INRA124 in Argentine and Bolivian cattle and revealed the migration and mobile pattern of the zebu gene in South America. Hanotte et al. (2000) investigated the Y-chromosome haplotype polymorphism of 984 individuals of 69 African local species in 22 countries of African Sahara by using INRA124 marker and showed that the haplotypes of taurine and zebu cattle were genetically different. Thus, INRA124 seems to be an indicator of taurine and zebu differentiation in various regions. Recently, Y-haplotypes have been used to analyze genetic diversity and origins of Portuguese cattle breed (Ginja et al., 2009). Y2 dominates in northern China (91.4\%), and Y3 dominates in southern China $(90.8 \%)$. Central China is an admixture zone, although there is an overall predominance of Y2 (72.0\%) (Li et al., 2013b). 
The objective of this study is to evaluate the polymorphism of Y-STR haplotypes in Chinese cattle populations and to understand their genetic relationship based on polymorphic analysis in different breeds.

\section{MATERIAL AND METHODS}

\section{Experimental animals and sample collection}

The Y-STR gene distribution in Chinese native cattle populations was investigated using 1016 healthy and heterogenetic males randomly selected from 9 typical native cattle breeds. In addition, 90 female cattle were randomly selected as control. About $15 \mathrm{~mL}$ blood samples were collected and placed in test tubes containing anticoagulant. The blood samples were transported to our laboratory at $4^{\circ} \mathrm{C}$ and stored at $-20^{\circ} \mathrm{C}$. The bull semen samples were collected and stored in liquid nitrogen.

\section{DNA extraction and polymerase chain reaction}

The DNA was extracted from the blood or semen by using Chelex-100 and proteinase K protocol (Walsh et al., 1991). The Y-STR gene polymorphism was studied by designing 6 sets of primers based on the gene sequences from http://www.ncbi.nlm.nih.gov of the National Center for Biotechnology Information (NCBI) and using the method of Liu et al. (2003); these primers were synthesized by Saibaisheng Biologic Company in Xi'an (Table 1). Polymerase chain reaction (PCR) amplification was carried out on a Geneamp 9700 thermal cycler (Biosystems) according to the manufacturer protocol. A total volume of $37.5 \mu \mathrm{L}$ PCR mixture contained $1 \mathrm{ng}$ genomic DNA, 1X GeneAmp PCR buffer (containing $1.5 \mathrm{mM} \mathrm{MgCl}$ ), $200 \mu \mathrm{M}$ dNTPs for each primer set, and 1.5 U Taq DNA polymerase. Thermal cycling was conducted using the following program: $95^{\circ} \mathrm{C}$ for $10 \mathrm{~min} ; 33$ cycles $\left(94^{\circ} \mathrm{C}\right.$ for $30 \mathrm{~s}, 59.2^{\circ} \mathrm{C}$ for $30 \mathrm{~s}$, and $72^{\circ} \mathrm{C}$ for $\left.45 \mathrm{~s}\right) ; 65^{\circ} \mathrm{C}$ for $45 \mathrm{~min}$; and the samples were held at $4^{\circ} \mathrm{C}$ until removal.

Table 1. Six Y-short tandem repeat (STR) locus names, designed primers, gene repeat type, fragment sizes, annealing temperature, and gene source for cattle Y-chromosome.

\begin{tabular}{|c|c|c|c|c|c|}
\hline Marker & Primer sequence $\left(5^{\prime}-3^{\prime}\right)$ & Type of repeat & Locus range (bp) & $\operatorname{Tm}\left({ }^{\circ} \mathrm{C}\right)$ & GenBank accession \\
\hline UMN0929 & $\begin{array}{l}\text { F: ACCAGCTGATACACAAGTGC } \\
\text { R: GGTCAGAGAATGAAACAGAG }\end{array}$ & $(\mathrm{CA})_{19}$ & $176-197$ & 61 & AF483749 \\
\hline UMN0108 & $\begin{array}{l}\text { F: GATACAGCTGAGTGACTAAC } \\
\text { R: GTGCAGACATCTGAGCTGTG }\end{array}$ & $(\mathrm{TG})_{18}$ & $\begin{array}{c}57-64 \\
189-250\end{array}$ & 60 & AF483744 \\
\hline UMN0920 & $\begin{array}{l}\text { F: GTTGAGGACTCTTGCATCTG } \\
\text { R: CACAGGCCTAGAAGATTGAG }\end{array}$ & $(\mathrm{TG})_{12}$ & $254-290$ & 58 & AF483763 \\
\hline INRA124 & $\begin{array}{l}\text { F: GATCTTTGCAACTGGTTTG } \\
\text { R: CAGGACACAGGTCTGACAATG }\end{array}$ & $(\mathrm{GT})_{4} \mathrm{~A}(\mathrm{TG})_{9}$ & $\begin{array}{c}58-67 \\
126-190\end{array}$ & 55 & X71546 \\
\hline UMN2404 & $\begin{array}{l}\text { F: GGTACAATTGAAAATATG } \\
\text { R: TGTACCTACACTGATATGTT }\end{array}$ & $(\mathrm{CA})_{18} \mathrm{~N}_{10}(\mathrm{TA})_{8}$ & $85-112$ & 54 & AF483769 \\
\hline UMN0103 & $\begin{array}{l}\text { F: ACACAGAGTATTCACCTGAG } \\
\text { R: ATTTACCTGGGTCAAAGCAC }\end{array}$ & $(\mathrm{CA})_{22}$ & $124-156$ & 60 & AF483757 \\
\hline
\end{tabular}

Electrophoresis was conducted using low-volume sequencing kits (DYCZ-24B). A total of $10.0 \mu \mathrm{L}(100 \mathrm{ng} / \mathrm{mL})$ PCR product was mixed with $2 \mu \mathrm{L} 6 \mathrm{X}$ bromophenol blue buffer solution and spotted on a $1.5 \%$ agarose gel. Following electrophoresis $(5 \mathrm{~V} / \mathrm{cm}$ for 0.5 to $1 \mathrm{~h})$, 
the gel was stained with $0.05 \%$ ethidium bromide. The DNA bands were viewed and photographed under ultraviolet light. The PCR products were then separated using $16 \%$ polyacrylamide gel electrophoresis at $4 \mathrm{~V} / \mathrm{cm}$ for $14 \mathrm{~h}$, and the DNA bands were silver stained again.

\section{Typing}

The products amplified at the 6 Y-STR loci were developed based on sequenced allelic ladder using Geneamp 9700 thermal cycler. Allelic ladders were constructed by combining all observed alleles from each locus.

\section{Quality control}

Quality assurance standards of all procedures are stipulated by the Scientific Working Group on DNA Analysis Methods (SWGDAM). Ten percent of the samples were randomly selected and genotyped twice using internal standards to further ensure analysis repeat and accuracy.

\section{Analysis of data}

Allele frequency and gene diversity (GD) were calculated using the Powerstat's method (Tereba et al., 1999; http://www.promega.com). Observed gene and haplotype diversities were calculated using the formula of Hou et al. (2001). The discrimination capacity was determined as the proportion of different haplotypes in the sample. Analysis of molecular variance (AMOVA) and population differentiation were performed using the ARLEQUIN software, version 2.000 (Schneider et al., 2000). A neighbor-joining tree was constructed from the distance matrix using the PHYLIP software to illustrate population relationships (Felsenstein, 1989).

\section{RESULTS}

\section{Y-STR haplotype polymorphism}

The designed PCR primers amplified Y-STR fragments ranging from 57 to $290 \mathrm{bp}$. All markers were also tested on female control samples, and all 6 gene loci displayed Y-chromosome specificity and polymorphism. The numbers of the 6 Y-STR alleles were 4, 5, 2, 2, 5, and 4 alleles at UMN0929, UMN0108, UMN0920, INRA124, UMN2404, and UMN0103 of 9 native cattle breeds, respectively (Table 2). Each breed has specific gene diversities in the 6 Y-STR loci, and their average genetic diversity value is 0.58 in 9 cattle breeds with the highest for Luxi cattle (0.65) and the lowest for Yanbian cattle (0.44). UMN0108 and UMN2404 were the most informative loci that had 0.73 gene diversity, while INRA124 was the least informative locus with 0.36 gene diversity. INRA124, UMN2404, and UMN0103 were identified as the diagnostic genes that distinguished $B$. taurus and B. indicus. The INRA124 gene has a 130 bp allele for $B$. indicus and a 132-bp allele for B. taurus. UMN2404 has 5 alleles, 2 of which were specific for $B$. taurus $(104,91 \mathrm{bp})$ and 3 were specific for $B$. indicus $(120,110$, and 85 bp). UMN0103 has 4 alleles specific for $B$. taurus (155, $140 \mathrm{bp}$ ) and B. indicus (136, $125 \mathrm{bp}$ ) (Kirikci et al., 2003; Karacaoren et al., 2008). 
Y.P. Xin et al.

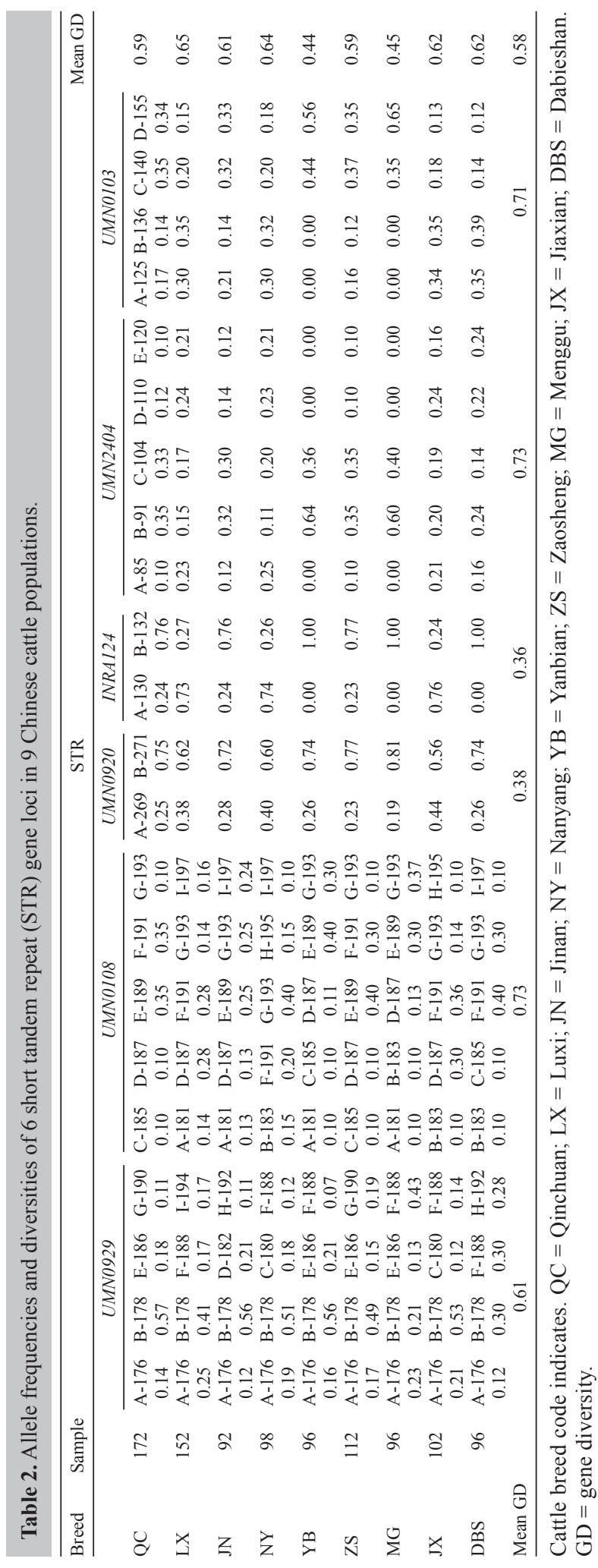


Y-STR haplotype showed an obvious diversity within and between breeds. A total of 302 haplotypes were identified from 1016 males of the 9 Chinese native cattle breeds, of which 186 were unique (Table 3). Luxi and Qinchuan cattle from the center of China have the most haplotype numbers (42 and 38, respectively), while the northern Yanbian and Menggu cattle have the least haplotype numbers, i.e., 29. The mid-southern and southern cattle have 29 to 35 haplotype numbers, respectively. This result showed a significant polymorphism in Chinese cattle populations. Further, each breed had 18-23 unique identification marker haplotype numbers. The frequencies of $B$. taurus gene alleles suggest that the northern Yanbian and Mengu cattle have $100 \%$ B. taurus decent, while Qinchuan, Zaoshen, and Jinnan cattle of central China have 68 to $92 \%$ B. taurus descent. The southern cattle contain less B. taurus decent, such as Jiaxian (25\%), Luxi (33\%), and Nanyang (34\%) cattle. The combined haplotype diversity of the 6 Y-STRs was between 0.90 and 0.98 . In general, their haplotype diversity was relative low within breeds, which indicates that these cattle breeds have a high genetic stability.

\begin{tabular}{|c|c|c|c|c|c|c|}
\hline Cattle breeds & Code & Sample size & No. of haplotype & Unique in the number & $\begin{array}{l}\text { Frequencies of } \\
\text { taurine alleles }(\%)\end{array}$ & Haplotype diversity \\
\hline Qinchuan & QC & 172 & 38 & 20 & 71 & $0.9045 \pm 0.0012$ \\
\hline Luxi & LX & 152 & 42 & 23 & 33 & $0.9675 \pm 0.0014$ \\
\hline Jinnan & $\mathrm{JN}$ & 92 & 28 & 21 & 68 & $0.9767 \pm 0.0022$ \\
\hline Nanyang & NY & 98 & 33 & 21 & 34 & $0.9686 \pm 0.0013$ \\
\hline Zaosheng & $\mathrm{ZS}$ & 112 & 34 & 20 & 92 & $0.9525 \pm 0.0031$ \\
\hline Jiaxian & JX & 102 & 34 & 22 & 25 & $0.9665 \pm 0.0015$ \\
\hline Dabieshan & DBS & 96 & 35 & 23 & 23 & $0.9544 \pm 0.0017$ \\
\hline Yanbian & YB & 96 & 29 & 18 & 100 & $0.9658 \pm 0.0024$ \\
\hline Menggu & MG & 96 & 29 & 18 & 100 & $0.9664 \pm 0.0018$ \\
\hline Total & & 1016 & 302 & 186 & 61 & $0.9581 \pm 0.0018$ \\
\hline
\end{tabular}

\section{T-STR genetic diversity across Chinese cattle breeds}

Analysis of molecular variance (Table 4) revealed that $98.76 \%$ genetic variance was from intra-population differences and $1.24 \%$ variation was from inter-population differences (fixation index $F_{\mathrm{ST}}=0.0124, \mathrm{P}<0.01$ ) in the 2 native $B$. taurus populations, i.e., northern Menggu and Yanbian cattle breeds. Further, $96.49 \%$ genetic variance was from intra-population differences and $3.51 \%$ was from inter-population differences (fixation index $F_{\mathrm{ST}}=0.0351$, $\mathrm{P}<0.01$ ) in the 7 mixed B. taurus and B. indicus populations such as Qinchuan, Luxi, Jinnan, Nanyang, Dabiashan, Zhaosheng, and Jiaxian cattle breeds. All these breeds contain B. taurus and $B$. indicus cattle blood, but various breeds have different amounts of taurine or indicine.

\begin{tabular}{|c|c|c|c|c|}
\hline Comparison & No. of cattle populations & Among cattle populations (\%) & Within cattle population (\%) & P value \\
\hline Bos taurus & 2 & 1.24 & 98.76 & $<0.01$ \\
\hline Bos indicus & 0 & - & - & $<0.01$ \\
\hline Bos taurus and Bos indicus & 7 & 3.51 & 96.49 & $<0.01$ \\
\hline
\end{tabular}

Bos taurus contains Yanbian and Menggu cattle breeds. Populations of mixed B. taurus and B. indicus contain the 7 cattle breeds-Qinchuan, Luxi, Jinan, Nanyang, Zaosheng, Dabieshan, and Jiaxian. 


\section{DISCUSSION}

\section{Y-STR haplotype polymorphism in the 9 Chinese native cattle breeds}

Y-STR was found on the male-specific Y-chromosome, and its coding genes were vital to male sex determination, spermatogenesis, and other male-related functions. The nonrecombinant Y-chromosome segment dictates the paternally inherited haploid transmission pattern. Y-STRs are polymorphisms among unrelated males and are inherited from the paternal line with little changes through generations. Y-STRs can be used to construct highly discriminative Y-haplotypes (Liu et al., 2002). Thus, the Y-chromosome-specific markers may be used in studies of animal or human origins and animal population genetics and variance. Our results showed that the 9 Chinese main cattle breeds showed Y-STR polymorphism in 6 loci, i.e., UMN0929, UMN0108, UMN0920, INRA124, UMN2404, and UMN0103. A total of 302 different haplotypes were identified from 1016 males of the 9 Chinese native cattle breeds, of which 186 were unique. Thus, these polymorphic loci have specificity for each cattle population. Interestingly, the polymorphism of these loci, especially, INRA124, UMN2404, and $U M N 0103$, suggests that the original history of Chinese cattle needs to be investigated to determine which cattle belong to $B$. taurus or B. indicus.

\section{Genetic relationship among Chinese cattle populations}

The data from the investigation of Y-STRs (INRA124, UMN2404, and UMN0103), in conjunction with the data published from the study of Y-chromosome morphologies of 262 males from 13 breeds (Liu et al., 2003), allowed the characterization of the distribution and genetic introgression of male indicine and taurine of Y-chromosome in Chinese native cattle. A phylogenetic tree was constructed based on Nei's distance from 13 cattle populations (Figure 1). This figure suggests that Dabieshan (DBS) vs Jiaxian (JX) cattle, and Qinchuan (QC) vs Jinnan (JN) cattle were first clustered together, which suggested that they had a very close genetic relationship. Meanwhile, Luxi (LX) and Nanyang (NY) cattle breeds were also included in this group with a short branch length. Finally, Zaosheng converged into a big family that consisted of major Chinese native yellow cattle populations from most regions of China. The other 2 main cattle populations such as Yanbian and Menggu cattle live in the north of China and belong to real native cattle breeds (B. taurus) that were not affected by the southern $B$. indicus cattle. Further, when the other 4 foreign cattle breeds were included in our phylogenetic tree, they obviously clustered into another group. This figure clearly showed a close genetic relationship of Chinese cattle populations. This result is consistent with the clustering nature of their mtDNA, blood protein polymorphism, geographic distribution, and breed evolution history.

\section{Y-STR haplotype geographical distribution in Chinese cattle populations}

The AMOVA results yielded interesting information on the internal history of the native cattle breeds. The genetic traces of the forced internal zebu migration displayed a movement from South towards North and East towards West of China. Our evidence of the population substructure in the paternal lineages of Chinese native cattle has implications for association studies, especially for the Y-chromosomal loci. 


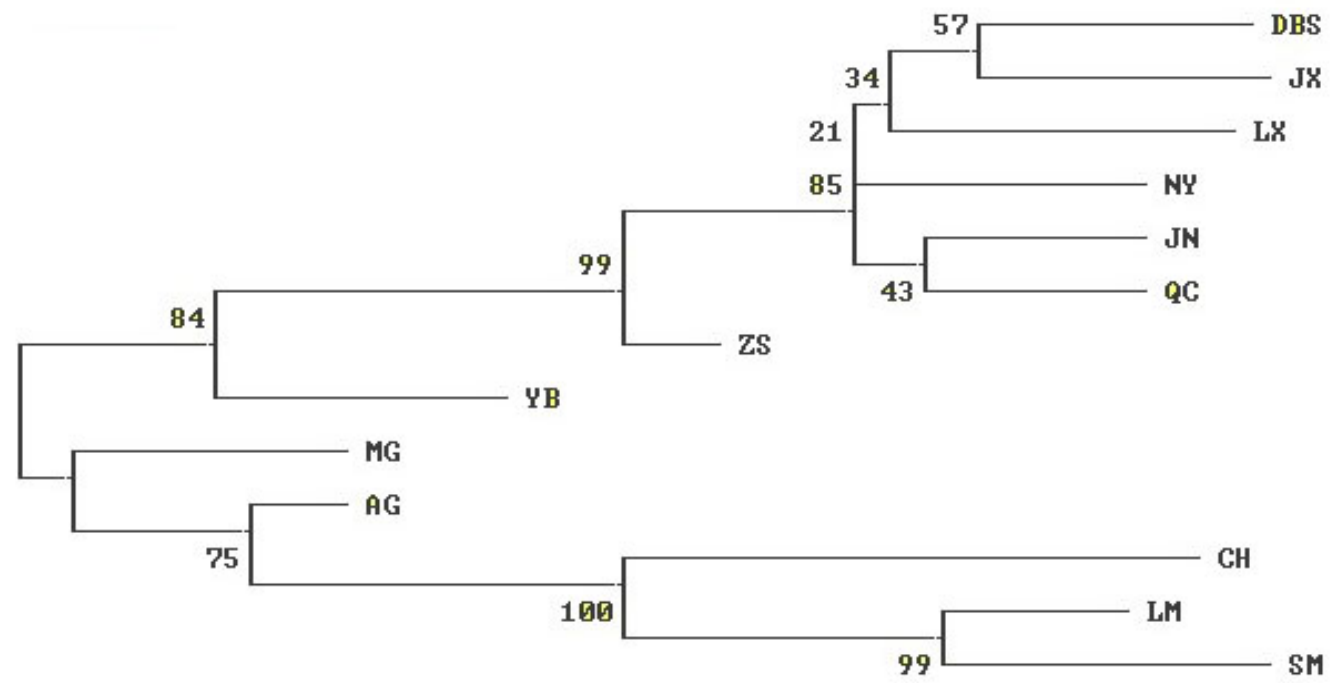

Figure 1. Phylogenetic tree developed based on Nei's distance from 9 Chinese cattle populations and other 4 foreign cattle breeds. Dabieshan (DBS) vs Jiaxian (JX) cattle and Qinchuan (QC) vs Jinnan (JN) cattle were first clustered together. Next, Luxi (LX) and Nanyang (NY) cattle were included in this group with a short branch length. Finally, along with Zaosheng (ZS) cattle, a big family of Chinese major native yellow cattle population from most regions of China was formed. The other 2 main cattle populations such as Yanbian (YB) and Menggu (MG) cattle live in the north of China and belong to real native cattle breeds (Bos taurus), which are affected by the southern Bos indicus zebu cattle, and were finally clustered into the Chinese cattle population. Further, when the other 4 foreign cattle breeds Angus (AG), Simmental (SM), Limousin (LM), and Charolais (CH) were included in our phylogenetic tree, they were obviously clustered into another group.

According to Y-STR haplotype gene frequencies and other studies on cell and molecular levels (Li et al., 2013a), a substructure proportion of B. taurus and B. indicus in Chinese cattle populations was calculated and a geographic distribution picture was profiled (Figure 2 ). The proportion of Y-chromosome haplotype of B. taurus decreases gradually, while that of $B$. indicus gradually increases from northern towards the southern area. The proportion of Y-chromosome haplotypes of B. taurus in Yanbian (YB), Tibetan (TB), Anxi (AX), and Mongolian (MG) cattle is $100 \%$; Zaosheng (ZS), QC, and Jinan $(\mathrm{JN})$ contains a little part of B. indicus blood. Bohai (BH), Nanyang (NY), LX, JX, Dabieshan (DBS), Xuanhan (XH), and Sinan (SN) cattle contain more $B$. indicus blood. Other southern cattle populations, including Ebian (EB), Wenling Hump (WL), Yunnan (YN), Diqing (DQ), Wannan (WN), Ji'an (JA), Minnan (MN), Leizhou (LZ), and Hainan (HN) cattle, almost did not contain Y-chromosome haplotypes of $B$. taurus. These cattle mainly belong to the $B$. indicus cattle group.

The geographical distribution of these Y-STR gene frequencies revealed a declining gradient of male indicine introgression from south towards north and east towards west in China. The highest frequency of taurine alleles $(93.0 \%)$ was found in the breeds from the northern group, while the highest percentage of indicine alleles $(93.0 \%)$ was observed in the southern cattle populations. The reason for the migration patterns might be due to historical events, geographical isolation, and climate difference. The frequencies of haplotypes of $B$. taurus of TB, AX, MG, YB, and ZS cattle are 100, 100, 100, 100, and 93.1\%, respectively. 
They mainly belong to $B$. taurus, which might be descendants of original Asian cattle. The frequencies of haplotypes of B. indicus of WN, JA, MN, LZ, HN, Sanjiang (SJ), EB, WL, and YN were the highest (100\%), and they mainly belonged to the zebu cattle system that might originate from or be affected by southern China or other countries, such as India or Malaysia. In the central and near northern areas, the mixing taurine and indicine Y-chromosome alleles appeared, and they showed a declining gradient of zebu introgression from East towards West with the indicine type in $75 \%$ of JX, $67 \%$ of LX, $66 \%$ of NY, $32 \%$ of JN, $29 \%$ of QC, and $6.9 \%$ of ZS cattle. The genetic influence of $B$. indicus in ZS, JN, and QC cattle was relatively less than that of JX, NY, and LX cattle. Jinnan region of Shanxi Province and the northwestern region of Shandong Province were also thought to belong to geographically converging areas of $B$. taurus and B. indicus. These data provided useful information for bull identification and paternity tests in Chinese native cattle.

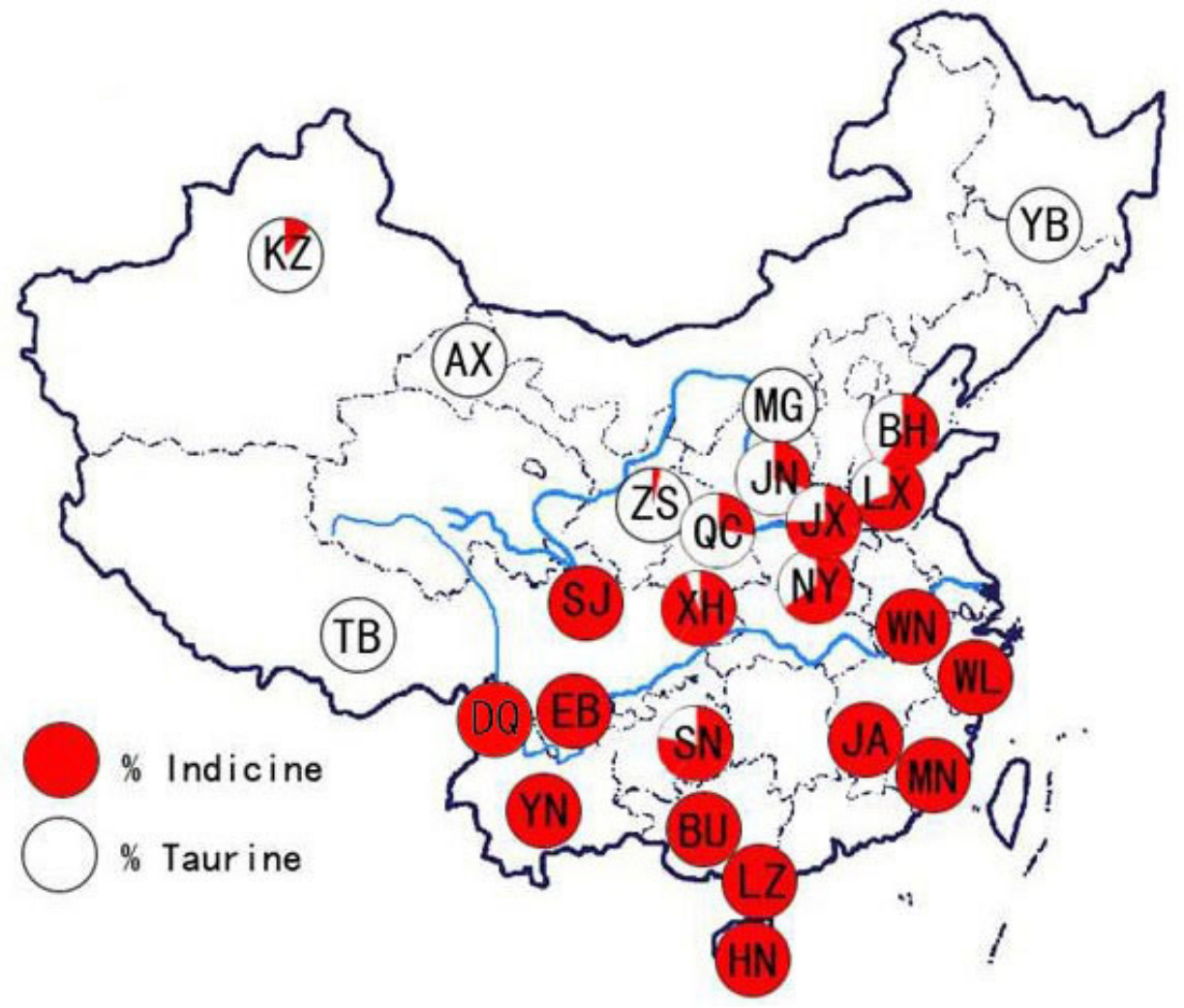

Figure 2. Geographic distribution of Chinese cattle populations and substructure proportion of Bos taurus (white) and Bos indicus (red) determined based on Y-chromosome haplotype analysis. The proportion of B. taurus decreases gradually, while that of $B$. indicus increases gradually from North towards South and from West to East. B. taurus in Yanbian (YB), Tibetan (TB), Anxi (AX), and Mongolian (MG) cattle is 100\%; Zaosheng (ZS), Qinchuan (QC), and Jinnan (JN) contain a little part of B. indicus blood. Bohai (BH), Nanyang (NY), Luxi (LX), Jiaxian (JX), Dabieshan (DBS), Xuanhan (XH), and Sinan (SN) cattle contain more B. indicus blood. Other southern cattle populations, including Ebian (EB), Wenling Hump (WL), Yunnan (YN), Diqing (DQ), Wannan (WN), Ji'an cattle (JA), Minnan (MN), Leizhou (LZ), and Hainan (HN) almost did not contain Y-chromosome haplotypes of $B$. taurus. These cattle mainly belonged to $B$. indicus cattle group. 
In summary, the Chinese native cattle breed is an excellent example to incorporate the Y-chromosomal data into existing knowledge of mitochondrial DNA variation and to reveal the entirely novel features of its population history. Our results suggest that Y-STRs in 6 loci show polymorphisms in Chinese cattle populations, with interesting genetic diversity. The Y-STR locus polymorphism, especially, INRA124, UMN2404, and UMN0103 loci, reveal Chinese cattle original history to determine which cattle belong to $B$. taurus or $B$. indicus. Most notably, a declining zebu introgression was exhibited from South to North and from East to West in Chinese geographical distribution, which implied that cattle population from various regions of China had been subjected to somewhat different evolution history.

\section{ACKNOWLEDGMENTS}

Research supported by the National "863" Program of China (\#XNY2013-8, \#2011AA100307, \#2013AA102505, \#CARS-38) and the National Natural Science Funds of China (\#31402044).

\section{REFERENCES}

Felsenstein J (1989). PHYLIP: Phylogeny inference package (Version 3.2). Cladistics 5: 164-166.

Gill P, Brenner C, Brinkmann B, Budowle B, et al. (2001). DNA Commission of the International Society of Forensic Genetics: recommendations on forensic analysis using Y-chromosome STRs. Forensic. Sci. Int. 124: 5-10.

Ginja C, Telo Da GL and Penedo MC (2009). Y chromosome haplotype analysis in Portuguese cattle breeds using SNPs and STRs. J. Hered. 100: 148-157.

Giovambattista G, Ripoli MV, De Luca JC, Mirol PM, et al. (2000). Male-mediated introgression of Bos indicus genes into Argentine and Bolivian Creole cattle breeds. Anim. Genet. 31: 302-305.

Hanotte O, Tawah CL, Bradley DG, Okomo M, et al. (2000). Geographic distribution and frequency of a taurine Bos taurus and an indicine Bos indicus Y specific allele amongst sub-saharan African cattle breeds. Mol. Ecol. 9: 387-396.

Hou YP, Zhang J, Li YB, Wu J, et al. (2001). Allele sequences of six new Y-STR loci and haplotypes in the Chinese Han population. Forensic. Sci. Int. 118: 147-152.

Jorde LB, Watkins WS, Bamshad MJ, Dixon ME, et al. (2000). The distribution of human genetic diversity: a comparison of mitochondrial, autosomal, and Y-chromosome data. Am. J. Hum. Genet. 66: 979-988.

Karacaoren B and Kadarmideen HN (2008). Principal component and clustering analysis of functional traits in Swiss dairy cattle. Turk. J. Vet. Anim. Sci. 32: 163-171.

Kirikci K, Zamani A and Durakbasi G (2003). A study on the chromosomes of Konya wild sheep (Ovis orientalis spp.): case report. Turk. J. Vet. Anim. Sci. 27: 281-283.

Li R, Zhang XM, Campana MG, Huang JP, et al. (2013a). Paternal origins of Chinese cattle. Anim. Genet. 44: 446-449.

Li R, Xie WM, Chang ZH, Wang SQ, et al. (2013b). Y chromosome diversity and paternal origin of Chinese cattle. Mol. Biol. Rep. 40: 6633-6636.

Liu WS, Mariani P, Beattie CW, Alexander LJ, et al. (2002). A radiation hybrid map for the bovine Y Chromosome. Mamm. Genome 13: 320-326.

Liu WS, Beattie CW and Ponce de Leon FA (2003). Bovine Y chromosome microsatellite polymorphisms. Cytogenet. Genome Res. 102: 53-58.

Redd AJ, Chamberlain VF, Kearney VF, Stover D, et al. (2006). Genetic structure among 38 populations from the United States based on 11 U.S. core Y chromosome STRs. J. Forensic. Sci. 51: 580-585.

Schneider S, Roessli D and Excoffier L (2000). Arlequin: A Software for Population Genetics Data Analysis. Ver 2.000. Genetics and Biometry Lab, Department of Anthropology. University of Geneva, Switzerland.

Tereba A (1999). Tools for Analysis of Population Statistics. In: Profiles in DNA. Vol. 2. Promega Corporation. 14-16.

Walsh PS, Metzger DA and Higuchi R (1991). Chelex 100 as a medium for simple extraction of DNA for PCR-based typing from forensic material. Biotechniques 10: 506-513.

Wu B (1988). Preliminary study on blood protein polymorphism and genetic relationship of Chinese yellow cattle breeds. J. Am. Vet. Sci. 25: 24-31. 